\title{
EL USO DE LA GAMIFICACIÓN CON PERSPECTIVA DE GÉNERO EN EL AULA: DIFICULTADES Y BENEFICIOS
}

\author{
O USO DA GAMIFICAÇÃO COM PERSPECTIVA DE GÊNERO NA SALA \\ DE AULA: DIFICULDADES E BENEFÍCIOS
}

\section{THE USE OF GAMIFICATION WITH A GENDER PERSPECTIVE IN THE CLASSROOM: DIFFICULTIES AND BENEFITS}

\author{
Pablo Soto Casás \\ Universidad de Santiago de Compostela, Espanha
}

\begin{abstract}
RESUMEN
El siguiente artículo se propone emplear el uso de la gamificación en el aula a través de una perspectiva de género. El contexto sociocultural cambia continuamente y, por lo tanto, las prácticas docentes tienen que evolucionar a la vez que la sociedad demanda cambios en esta época de empoderamiento de grupos sociales discriminados. Toda esta situación entre en fricción con el desamparo educativo que ofrecen los currículos nacionales de educación ya que no amparan al profesorado a la hora de enfrentarse a esta desconexión sociocultural con el alumnado. Para solucionar este problema se propone el uso de la gamificación en el aula para poder conectar la práctica docente con el universo social del alumnado. A lo largo de este artículo se desarrollarán diversos conceptos sobre qué es lo que significa gamificar, en que influye en la práctica docente y de qué manera se puede implementar en las unidades didácticas. Para concluir, se analizarán los componentes característicos de las industrias culturales y se elaborará una interpretación de como estos productos provocan un sesgo de género y como se puede adquirir el mensaje del medio para su uso en el aula gamificada.
\end{abstract}

Palabras clave: Gamificación. Metodología. Videojuegos. Género.

\section{RESUMO}

O artigo a seguir tem como objetivo empregar o uso da gamificação na sala de aula através de uma perspectiva de gênero. $O$ contexto sociocultural muda continuamente e, portanto, as práticas de ensino precisam evoluir à medida que a sociedade exige mudanças nesta era de empoderamento de grupos sociais discriminados. Toda essa situação entra em conflito com o desamparo educacional oferecido pelos currículos nacionais de educação, uma vez que eles não protegem os professores quando se trata de lidar com essa desconexão sociocultural com os alunos. Para solucionar esse problema, propõe-se o uso da gamificação em sala de aula, a fim de conectar a prática de ensino ao universo social dos alunos.

\begin{tabular}{|l|l|l|l|l|l|}
\hline Revista RBBA & ISSN 2316-1205 & Vitória da Conquista & V. 9 n o 1 & p. 79-96 & Julho/2020 \\
\hline
\end{tabular}


Ao longo deste artigo, vários conceitos serão desenvolvidos sobre o que significa gamificação, como ela influencia a prática de ensino e como ela pode ser implementada em unidades de ensino. Para concluir, os componentes característicos das indústrias culturais serão analisados e será feita uma interpretação de como esses produtos causam um viés de gênero e como a mensagem do meio pode ser adquirida para uso em sala de aula gamificada.

Palavras-chave: Gamificação. Metodología. Videogame. Gênero.

\begin{abstract}
The following article aims to employ the use of gamification in the classroom through a gender perspective. The socio-cultural context changes continuously and, therefore, teaching practices need to evolve as society demands changes in this era of empowerment of discriminated social groups. This whole situation conflicts with the educational helplessness offered by national education curricula, since they do not protect teachers when it comes to dealing with this sociocultural disconnect with students. To solve this problem, it is proposed to use the gamification in the classroom, in order to connect the teaching practice to the students' social universe. Throughout this article, several concepts will be developed about what gamification means, how it influences teaching practice and how it can be implemented in teaching units. To conclude, the characteristic components of cultural industries will be analyzed and an interpretation will be made of how these products cause a gender bias and how the media message can be acquired for use in the gamified classroom.
\end{abstract}

Keywords: Gamification. Methodology. Video game. Gender.

\title{
Introducción
}

La perspectiva de género ha cobrado importancia tanto en el discurso político como en la interpretación de la realidad. La influencia de esta perspectiva trabajada desde los estudios de género y el feminismo han calado en la sociedad y, por lo tanto, la escuela, como institución social, también se ha visto influenciada por este campo. No obstante, tal y como voy a explicar en este artículo, la elaboración de materiales educativos a través de esta perspectiva presenta varios problemas para el profesorado por varios motivos.

Para empezar, una de las principales causas de esta problemática es el proceso de la dinámica social. Esta consecuencia del desarrollo social se puede clasificar como el proceso de evolución social que provoca diferencias significativas intergeneracionales. Por ejemplo, si se hace una comparación entre las características de las necesidades educativas entre la generación nacida en el 2000 y la que ha nacido en 2010 se pueden observas claras diferencias, ya sea a través de las necesidades que surgen a través de los hábitos de consumo de carácter neoliberal del momento, las cuales determinan la construcción identitaria del alumnado, o simplemente las condiciones socioeconómicas que marcan las necesidades del momento.

Revista RBBA Revista Binacional Brasil Argentina 


\section{EL USO DE LA GAMIFICACIÓN CON PERSPECTIVA DE GÉNERO EN EL AULA: DIFICULTADES Y BENEFICIOS}

Otra medida a tener en cuenta sería la sistematización de la educación a través del currículo nacional. Ya que ésta provoca una asimetría simbólica generacional entre el alumnado y el profesorado debido al sesgo producido por la rigidez característica del sistema educativo, tanto por no tener en cuenta los grandes los cambios que ocurren en la sociedad a corto plazo, como por la carencia en la adquisición de la simbología necesaria para elaborar una comunicación adecuada al contexto sociocultural del alumnado.

En consecuencia, este proceso de inadaptación social es legitimado por actores institucionales junto al rol de la figura del profesorado, ya que no adaptan sus contenidos curriculares a la situación social del alumnado debido a la brecha generacional, mencionada anteriormente, que se produce por la evolución del contexto sociocultural de las tecnologías.

Por lo tanto, el papel del docente se vuelve complejo a la par de arduo a la hora de implementar metodologías vanguardistas debido a su escasa adaptación al medio tecnológico. Esto está causado por la formación necesaria que se necesita para impartir este tipo de medidas es realmente escasa y, además, requiere un esfuerzo y motivación personal por parte del profesorado que no todo el mundo está dispuesto a compartir.

Para ello, una de las posibles soluciones que surge para las adaptaciones curriculares correspondientes a la realidad social viene dada por la adecuación de la realidad conocida del alumnado, es decir, elaborar una propuesta basada en los productos culturales que consumen y que, por lo tanto, le proporcionan conocimiento de la realidad social del momento. Un ejemplo claro para escenificar esta perspectiva serían los videojuegos, ya que son un medio que les nutre con un entramado de conocimientos simbólicos y culturales de actualidad. No obstante, estos productos provocan ciertas discriminaciones, tales como:

Proporcionan un conocimiento cultural sesgado por su carácter globalizador.

Producen brechas económicas por las dificultades tecnológicas y económicas a la hora acceder a ellos.

Muchas de sus narrativas están vinculadas al propio medio lenguaje audiovisual del medio y no se representan diversidad en los modelos de género.

Sin embargo, el uso de las características que proporcionan estas experiencias en el aula son un elemento a tener en cuenta para desbloquear los desafíos que propone tanto la sociedad como el alumnado.

Por lo tanto, en este artículo se propone la aplicación de la gamificación como una herramienta metodológica en la intervención de los problemas que presenta el aula actualmente.

Revista RBBA Revista Binacional Brasil Argentina 
Muchas de las características que ofrecen los juegos y, además, los videojuegos pueden ofrecer un aire innovador en el proceso de construcción de las unidades didácticas. Para poder desarrollar esta afirmación se definirán este término y en los siguientes párrafos se explicarán los beneficios y desventajas que tiene la implementación de esta modelo en el aula, como también la pauta de aplicación en el aula a través de una perspectiva de género.

\section{La impregnación de la cultura en la realidad de la persona mediante la adquisición de la interacción entre los Imaginarios Sociales en las Representaciones Sociales.}

El proceso de construcción social de la persona ocurre desde el primer momento de vida. A través del uso del aprendizaje simbólico el ser humano es capaz de establecer pautas de comunicación entre iguales mediante el uso de la herencia simbólica adquirida a lo largo de su vida. No obstante, este corpus adquirido forma parte de un conjunto de elementos que han sido predefinidos por sus antepasados y, por lo tanto, forman parte de un entramado cultural que ha sido definido previamente (Schutz, 2015, pp.41). Por ello, la construcción cultural es un punto clave para entender la interpretación de la realidad construida.

Desde la perspectiva de los Imaginarios Sociales (PINTOS, 2014) se puede entender como estos conceptos cobran sentido en nuestra imaginación y forman esquemas vivos de elementos preconceptualizados. No obstante, estos elementos están situados en la sociedad, son los llamados Representaciones Sociales. Este término tiene por definición ser un mecanismo con el que se configuran los imaginarios sociales y, por lo tanto, éstos son marcos de referencia desde los cuales los sujetos decodifican las representaciones que le vienen del contexto y las hacen suyas (PÉREZ, 2017). Por ejemplo, Aarseth propone analizar la figura del dragón en las narrativas. Él considera al dragón como un producto elaborado a través del tiempo y que ha sufrido variaciones en su significado de la representación de este elemento en los productos culturales. "[...] there would have been no difference between our experience of Tolkien's world and the world of EverQuest. One dragon is clearly fictional, but the other is simulated." (AARSETH, 2008, p.2).

Con este ejemplo se afirma que la figura imaginada del dragón se establece en la sociedad mediante su aparición en las Representaciones Sociales, (MOSCOVICI, 1977 en MORA, 2002, AARSETH, 2008, PLAZA 2017), las cuales constituyen un entramado tanto artístico como estructural, que les permiten a las personas la posibilidad de interactuar con el 
mundo. Concretamente, Aarseth menciona estas dos obras influyentes a través de dos perspectivas, siendo la primera desde el universo del Señor de los Anillos a la segunda que sería al universo planteado en el videojuego EverQuest. En esta comparación hay una gran diferencia entre lo que significa el dragón y como está representado. Estas diferencias son significativas, ya que la influencia de las obras principales crea un corpus cultural que se reproduce y evoluciona la constitución de los Imaginarios Sociales junto a la representación de ellos en las obras. No obstante, estas representaciones probablemente difieran con la forma imaginada del dragón según cada indivíduo, ya que las adquisiciones culturales son distintas según los procesos de construcción individual y por lo tanto, las formas de representación serán distintas según la experiencia individual.

En consecuencia, los imaginarios sociales entran en fricción con las representaciones sociales por la individualidad única que presenta el individuo en el proceso de comunicación e interpretación simbólica. Por ende, el proceso de sistematización de la educación en el aula tiene que ser adaptada mediante la creación de una narrativa única para poder construir de manera uniforme la adquisición de conocimientos y competencias a trabajar. Estas aportaciones hacen que se produzcan dificultades a la hora de aplicar nuevas metodologías en el aula son obvias. Tal y como ya hemos hablado anteriormente, la adquisición de entramado simbólico renovado entra en conflicto con los problemas que surgen en la aplicación en nuestro discurso cotidiano. Debido a esto, una solución que se puede proporcionar a la realidad social del aula puede ser a través de la gamificación, pero este término produce várias preguntas, desde ¿qué ventajas y desventajas proporciona?, ¿cuáles son las pautas de aplicación?, ¿necesito cumplir algún perfil en concreto para aplicar esta metodología? Antes de empezar a discutir sobre la gamificación y sus consecuencias, se hablará acerca de los distintos significados de la gamificación y que diversidad de situaciones puede proporcionar este término.

\section{La aplicación de la evolución tecnológica en educación, la transición del edutainment a los serious games.}

Desde los primeros usos de las tecnologías en educación a tal y como estamos hoy en día ha transcurrido mucho, desde los primeros comienzos del uso de juegos a través del “edutainment", a la transición provocada con la elaboración de los "serious games", junto a la 
innovación metodológica elaborada a través de la gamificación, el proceso de adaptación curricular en el ámbito educativo ha ido evolucionando a lo largo del tiempo.

El comienzo de la aplicación tecnológica en el ámbito educativo surgió por primera vez a través del "edutainment". Muchas de las primeras aplicaciones que se realizaron dentro de esta opción surgieron con el fin de enseñar al alumnado conocimientos, habilidades y conceptos básicos para la introducción al alumnado a ciertos campos de estudio. En un contexto educativo donde se necesitaba una enseñanza basada en el aprendizaje de habilidades, el "edutainment" no cumplía las expectativas propuestas porque los resultados finales este complemento de enseñanza lograba una escasa evolución del alumnado a la hora de adquirir conocimientos, tales como, mejoras en el entendimiento o ayudar a evaluar el aprendizaje del alumnado (CHARSKY, 2010).

Con este proceso de evolución metodológica a la par que tecnológica, se elaboró una mejora sustancial basada en la elaboración de juegos educativos. Las diferencias significativas que surgen entre el "edutainment" y los "serious games" se traducen a través del cambio de enfoque que se elaboran a través de éstos últimos. Los "serious games" se caracterizan por proporcionar a la persona videojugadora habilidades basadas en un pensamiento crítico a través de las características del juego. Se tiene el ejemplo de páginas como Itch.io proporcionan un diverso catálogo de "serious games" hechos por una amplia variedad de gente aficionada y pequeñas empresas dedicadas a promocionar sus productos.

No obstante, estas empresas y usuarios al tener una menor cantidad de dinero para invertir en el desarrollo de sus juegos, el producto final, si se compara con el de una desarrolladora multinacional, como Microsoft, Ubisoft, etc., va a ser peor, tanto gráficamente, como jugabilísticamente. Provocando así una menor atracción del alumnado por este tipo de juegos.

Por otro lado, también se tiene a disposición juegos de simulación tales como Civilization, SimCity. Ya que, estos productos tienen características similares con los tipos de juegos mencionados anteriormente, porque mezclan funciones propias del juego con la capacidad de aprendizaje del alumnado. (CHARSKY, 2010) No obstante, este tipo de juegos tienden a ser calificados como aburridos por parte de los jugadores por la falta de recursos que se tienen a la hora de producir.

Para solucionar este problema, en este artículo se propone como solución a las necesidades que surgen a través del alumnado, junto a los problemas que el edutainment y los 
serious games tienen, la metodología basada en la gamificación del aprendizaje. Pero ¿Qué es la gamificación? Y, sobre todo, ¿Cómo se elabora?

\section{Gamificar como metodología educativa. Definición, ventajas y contras.}

El término gamificación ha estado en el debate académico en la última década, de hecho, todavía es un debate ferviente en el ámbito educativo ya que la aplicación de esta metodología provoca una multitud de pensamientos respecto a ella. Desde el ámbito de académico se dice que la adquisición masiva de datos mediante el aprendizaje memorístico es contraproducente, por ello, el uso del juego como elemento de aprendizaje es una posibilidad para tener en cuenta.

No obstante, esta propuesta metodológica no es nueva, ya se hablaba del constructivismo a través del juego para favorecer el aprendizaje de habilidades, pero, si la gamificación tiene tantas similitudes con los pensamientos de Piaget, entonces ¿cuáles son las diferencias de la gamificación frente las metodologías constructivistas? Las principales características que la gamificación tiene están basadas en la estructuración de los contenidos en base a una narrativa que articula la disposición tanto del alumnado como del profesorado, junto al tratamiento de los contenidos que componen la materia en base a una metodología de aprendizaje cooperativo.

A pesar de esta conceptualización, en la academia se ha trabajado diferentes posibilidades para definir esta metodología en base al resultado que han obtenido en el aula debido a la complejidad de implementación que esta metodología presenta. Mucho personal del ámbito académico argumenta que la gamificación se basa sencillamente en una metodología basada en la obtención de puntos para conseguir medallas cuando se adquiere un determinado progreso, al igual que el uso de un castigo cuando el trabajo se traduce en un comportamiento poco adecuado. (LEE Y HAMMER, 2011), otras figuras de prestigio argumentan que son el resultado de la aplicación de elementos del juego a la metodología (DETERDING et al., 2011) con el fin de lograr una adquisición de un compendio de experiencias útiles para el usuario.

Aunque, estas definiciones anteriores solo operan con la experiencia de la aplicación metodológica, hay varias definiciones de gamificación que se orientan hacia mejorar la experiencia del usuario, dentro de este enfoque se puede definir el término gamificación como el proceso de utilizar mecánicas del juego para promover el aprendizaje con el fin de aumentar 
la motivación y enganchar al usuario en las narrativas para resolver los problemas propuestos por el profesorado (KAPP, 2012, ZICHERMANN Y CUNNINGHAM, 2011).

Sin embargo, lo dicho anteriormente solo valora los aspectos positivos acerca del uso de esta práctica. Tal y como hemos descrito, la gamificación es la adquisición de conocimientos y experiencias a través de la implementación de las características del juego en procesos caracterizados por ser no lúdicos, no obstante, hay varias autoras y autores que valoran esta metodología como una imposición de las características del mercado neoliberal del momento en las metodologías educativas. Dentro de este ámbito tenemos a Fuchs (FUCHS et al., 2014, p.8) y a su grupo de investigadores que argumentan que la gamificación es una práctica promovida por gurús del marketing y diseñadores y a Ian Bogost (2014) que califica a la gamificación como "gamification is bullshit" con un análisis de sus dos componentes siendo "game-" como una fuente de atracción y su sufijo "-ification" como proceso de obtener algo de manera fácil y sencilla (CRAWFORD Y MURIEL, 2018).

Después de ver estas definiciones podemos observar que hay fuertes discrepancias entre las diversas definiciones tras definir a la gamificación. Con todo este entramado de conocimiento se puede sacar una cosa clara y es que el éxito de la implementación de esta metodología se encuentra bajo el papel del profesorado para adaptar el ritmo y la aplicación de la experiencia diseñada.

\section{El aprendizaje a través de la narrativa adquirida en la gamificación, cómo implementarla y qué sesgos produce.}

Una vez conocidas las diferencias que surgen entre la implementación de los "serious games", el "edutainment" y la gamificación, se va a explicar a continuación cuáles son las principales características que se tienen que tener en cuenta a la hora de implementar esta metodología en el aula. Dentro de los diferentes componentes de la gamificación podemos mencionar tres principales características, las cuales serían las mecánicas, las dinámicas y las percepciones.

\section{Las mecánicas}

Vamos a empezar a definir el concepto de mecánicas dentro de la gamificación. Para Labrador (2020) las mecánicas de juego se pueden definir como las reglas que limitan las Revista RBBA $\mid$ Revista Binacional Brasil Argentina 


\section{EL USO DE LA GAMIFICACIÓN CON PERSPECTIVA DE GÉNERO EN EL AULA: DIFICULTADES Y BENEFICIOS}

acciones de las personas que participan en el juego. Estas opciones provocan una serie de pautas de acción definidas en la previsión de comportamiento a través de la preparación del juego. Por lo tanto, la elección de las distintas mecánicas escogidas para la elaboración del juego influirá tanto la forma de jugar como la interacción entre las personas participantes en el juego.

Para empezar a definir las diferentes variables que hay en las mecánicas de juego vamos a centrarnos dentro de la agrupación que menciona Labrador (2020). Según su investigación las mecánicas se aglutinan respecto a varios tipos, él enumera una serie de mecánicas que están caracterizadas con diversos ejemplos de ellas.

Mecánicas de progreso y evolución o Niveles: la implementación de estas mecánicas se basa en elaborar un sistema de ranking según las necesidades que la narrativa implementada necesite, ya sea mediante una distribución de puntos o establecer un sistema de puntuación, mediante la adquisición de medallas o incluso obtener recompensas según los logros alcanzados.

Mecánicas de colección o Recompensas: ya sean a través de una repartición fija o aleatoria de diversas mejoras en el arsenal del alumnado a través de la asignación de equipamiento, como monedas virtuales, o incluso la dotación de huevos de pascua.

Mecánicas de personalización: a través de la modificación de avatares predefinidos el alumnado participante en la actividad podrá modificar la apariencia de algunas partes del mundo propuesto.

Mecánicas de socialización o área social: este tipo de mecánicas nos permite elaborar una adecuada distribución por equipos manteniendo una paridad (si es posible) dentro del alumnado, o incluso elaborar unos gremios o clanes según la orientación narrativa propuesta. A su vez, se puede implementar un chat para que las otras facciones o grupos tengan una forma de poder comunicarse entre sí.

Mecánicas de presión o Presión por tiempo: son aquellas mecánicas que se implementan con el fin de añadir algún desafío épico, o incluso algún evento especial con el fin de añadir un énfasis en la trama.

Mecánicas de expresión o Dibujar: desde una perspectiva narrativa, performativa e interpretativa la capacidad de inmersión en el rol diseñado puede provocar una mejora en la inmersión del alumnado dentro de la narrativa propuesta.

Mecánicas de avance (en el espacio o el tiempo) o Puntos de acción: estas mecánicas rigen la posibilidad de los participantes respecto a la habilidad relaciona respecto a los Revista RBBA Revista Binacional Brasil Argentina 


\section{EL USO DE LA GAMIFICACIÓN CON PERSPECTIVA DE GÉNERO EN EL AULA: DIFICULTADES Y BENEFICIOS}

movimientos/desplazamiento, si hay alguna posibilidad de movimiento oculto, si se proporciona algún ítem de acceso, o incluso algún tutorial para enseñar ciertas habilidades que posibiliten el avance del alumnado en la trama, o incluso tener la posibilidad de obtener un salvavidas ya sea para pedir pistas y evitar atascos en el desarrollo del proceso.

Mecánicas de acción o Actuar: son todas aquellas acciones que rigen la interacción del alumnado con el medio presentado, ya sea a través de la programación de ciertas acciones, subastas, como el resultado de la compra/venta de objetos o incluso la posibilidad de hacer duelos, obtener la petición de búsqueda de algún objeto, poder disparar, subir el nivel de las habilidades.

Mecánicas de condiciones de victoria o Mayoría: estas mecánicas ofrecen una adecuación en la consecución de objetivos a través de puntos, obtención de terrenos, o, por otra parte, la posibilidad de resolver acertijos o puzles propuestos, hasta determinar la victoria, ya sea llegando el primero o consiguiendo los objetivos propuestos.

Como se puede apreciar la elección de elementos definitorios de juego es un elemento clave a la hora de planificar la gamificación, además, es de vital importancia que todas las mecánicas tengan un sincronización e interacción armoniosa entre ellas y, sobre todo, una cohesión mutua con respecto a los objetivos propuestos en el diseño de la unidad didáctica.

No obstante, estas mecánicas están orientadas a la consecución de objetivos y la finalidad de estas es la adquisición de aprendizajes y de competencias clave. Pero es aquí donde el sesgo social del docente surge a través de los imaginarios sociales, ya que muchos de los ejemplos de implementación actuales indudablemente tendrán sesgos de género por los esquemas mentales que están impregnados por el contexto sociocultural del momento. Está claro que si la sociedad en la que vivimos es machista las enseñanzas y los esquemas mentales que surgen a través de los imaginarios se asentarán en nuestro pensamiento y modificará la percepción de la realidad, tergiversando así la visión hacía una orientación sexista en la elaboración de contenidos docentes.

En cuanto a la implementación de estas mecánicas las dificultades que surgen a través la gamificación no son pocas, ya que son muchos elementos a tener en cuenta a la hora de elaborar la unidad didáctica y, además se tiene que valorar desde una óptica de género la elaboración de las actividades con el fin de evitar sesgos de género en la actividad propuesta.

Desde este punto de vista hay mecánicas que suponen una dificultad añadida a la hora de diseñar las actividades, como, por ejemplo, tal y como pueden suceder en las mecánicas de Revista RBBA Revista Binacional Brasil Argentina 
socialización, en las mecánicas de expresión o incluso en las mecánicas de acción o actuar. Ya que, por lo general, estas mecánicas se componen por una fuerte influencia por lo social y, además, tienden a tener un claro sesgo de género por todos los imaginarios sociales vinculados a los productos culturales y a la narrativa sociohistórica que se han ido heredando culturalmente.

Por lo tanto, la consecución de las actividades propuestas en la actividad gamificada corre a cargo del personal docente, ya que está en su mano evitar sesgos de género en el grupo, y no solo eso, sino que también tendrá que tener sumo cuidado a la hora de planificar las actividades propuestas, ya sea salvaguardando la diversidad de los personajes representados en la narrativa, como también dentro de la interacción entre el mundo construido y el alumnado con el fin de preservar el bienestar de las dinámicas grupales.

\section{Dinámicas}

Según Labrador (2020) se pueden definir las dinámicas como las acciones que realizan los jugadores cuando ejecutan las reglas establecidas. Las dinámicas tienen por característica ser la una de las partes más complejas a la hora de diseñar el juego, ya que este concepto depende de las mecánicas implementadas, y, por lo tanto, cualquier variación que surja en el diseño de la actividad puede cambiar por completo la inmersión del alumnado en las actividades propuestas.

Por lo tanto, es de vital importancia realizar varias veces comprobaciones para verificar la efectividad de los cambios y las nuevas propuestas de intervención en el diseño de actividades. Estos cambios en el diseño de las actividades son necesarios para poder elaborar una actividad adecuada basada en la interacción de las personas. En consecuencia, cabe destacar la importancia de las técnicas basadas en conocer los procesos de Experiencia de usuario, las llamadas UX (User experience) ya que permiten recopilar una serie de respuestas producidas por el usuario en torno a la experiencia que ha tenido a lo largo de la propuesta de intervención.

Muchos de estos mecanismos de evaluación sobre la experiencia de usuario han ido evolucionando y variando a lo largo del tiempo, en este artículo vamos a mencionar el "Design Thinking" justificando la elección de esta modalidad por la empatía natural que surge a través de implementación de esta técnica UX. Esta técnica está argumentada por Labrador (2020), él clasifica cada uno de los componentes del "Design Thinking” en:

Comprender las necesidades de los usuarios: el papel del profesorado en los procesos de creación de contenidos es imprescindible, ya que cada unidad didáctica propuesta Revista RBBA $\mid$ Revista Binacional Brasil Argentina 
necesita adaptaciones curriculares para poder llevarse a cabo. La habilidad que se necesita aquí es la empatía requerida para entender las circunstancias en las que se ve envuelto el alumnado. Una vez establecida esta parte se puede proceder al siguiente paso.

Definir los puntos relevantes: a partir de la elaboración de cuestionarios y entrevistas en profundidad se pueden extraer los puntos más relevantes a la hora de implementar nuevas dinámicas en el aula. Una vez adquiridas las técnicas de indagación con usuarios, se extraen los puntos más relevantes y significativos de los que se van a constituir la innovación curricular.

Idear: una vez clarificadas las necesidades esenciales de los distintos usuarios, comienza la elaboración de la construcción de la unidad didáctica. El objetivo de esta etapa es elaborar una lista de diferentes opciones con el fin de desarrollar prácticas que desarrollen los objetivos propuestos en el currículo nacional y que, a su vez, respondan a las necesidades del alumnado.

Prototipar: una vez ideado el modelo a seguir, es necesario hacer una prueba previa para poder obtener datos sobre las ideas predefinidas con anterioridad. Este es un proceso clave para evaluar nuestra práctica docente y averiguar si se han cometido errores a la hora de aplicar la perspectiva de género en las actividades propuestas. Para ello, es necesario realizar várias pruebas con grupos diversos para así de obtener una variedad de resultados.

Testear: Una vez desarrollada el primer prototipo de actuación se procederá a probar el producto en várias condiciones en el aula hasta conseguir un resultado positivo y que no discrimine a la diversidad del alumnado por su contenido.

Una vez clarificada la forma de evaluar y elaborar el funcionamiento de las mecánicas a través del análisis de la experiencia del usuario, queda por nombrar la última parte del desarrollo de la gamificación, la percepción del usuario.

\section{Percepciones}

La percepción dentro de la definición que propone Labrador (2020) se compone dentro de "los sentimientos y emociones que emergen en el jugador cuando al ejecutar las mecánicas realiza (o los demás realizan) acciones. Dentro de las percepciones del jugador que surgen a través del juego se pueden clasificar en:

Sensaciones: siendo esta la forma que tiene el jugador de experimentar la situación propuesta a través de los sentidos

Fantasía: ocurre cuando el juego crea imágenes en la cabeza de la persona jugadora. 
Narrativa: sucede cuando el juego presenta historias hacen ejercitar al jugador la empatía frente al personaje, mundo y sistema propuesto.

Reto: se establece cuando la persona videojugadora recurre a las herramientas propuestas por el sistema frente a un desafío programado.

Amistad: es la unificación del grupo a través del juego elaborado.

Descubrimiento: dentro de la estimulación que surge a través del clima y la atmosfera presentado por la actividad propuesta, el alumnado encontrará este espacio creado como un estímulo y descubrir esta parte innovadora explorar el mundo propuesto en el juego.

Expresión: las dinámicas propuestas tienen como resultado una exaltación de la forma de comunicarse entre el alumnado, provocando así un refuerzo cognitivo en cuanto a la promoción en la creación del sentimiento identitario grupal.

Integración: la implementación de las características del juego logra provocar a la persona jugadora una unión plena, proporcionando una sensación única.

Con el fin de obtener una sensación armónica entre motivación y las emociones el desarrollo de los elementos perceptivos mencionados anteriormente es clave para alcanzar la experiencia gamificada. La manera de conectar el aprendizaje junto a la narrativa creada se basa en enganchar al alumnado con las mismas características que otros productos culturales ofrecen, como por ejemplo los videojuegos.

No obstante, el proceso de gamificación de una unidad didáctica no implica que el desarrollo metodológico se ciña solamente a lo lúdico, ya que no se debe descuidar el objetivo de esta actividad que es la adquisición valores, habilidades y competencias básicas.

Por lo tanto, para representar lo dicho anteriormente, vamos a explicar a continuación cuáles son las características de los juegos presentados en la conferencia del E3 de 2019 y para así conocer cuál es la situación del mercado en este momento con el fin de mostrar de qué manera se pueden obtener elementos de las narrativas producidas en los videojuegos.

\section{Los sesgos de género en los productos culturales del momento}

Muchas de las recomendaciones que se han dicho en este artículo a la hora de implementar un sistema gamificado en el aula se corresponden a abstraer ciertas características de los videojuegos con el fin de aplicarlo en el aula y así resolver la brecha generacional 


\section{EL USO DE LA GAMIFICACIÓN CON PERSPECTIVA DE GÉNERO EN EL AULA: DIFICULTADES Y BENEFICIOS}

mencionada anteriormente con respecto a la elaboración de contenidos atrayentes para el alumnado.

No obstante, estos productos culturales tienen ciertas características en común y es que el propio medio está sesgado. Para evaluar el sesgo de género en la industria se procederá a observar el análisis del equipo de Anita Sarkeesian (Figura 1) en su evaluación anual de la huella de género en la industria del videojuego a través de un análisis del E3 de 2019 (Electronic Entertaiment Expo). Esta conferencia es conocida por ser el expositor anual donde se muestra que videojuegos serán lanzados en los siguientes años por las grandes empresas multinacionales y, por lo tanto, está conferencia marcará tanto la tónica, como la temática de los juegos presentados por la industria en los próximos años. Tal y como se puede apreciar en el gráfico de abajo, hay una gran diferencia entre los juegos protagonizados por personajes femeninos (5\%), masculinos (22\%) y LGTBIQ+ (2\%).

Figura 1: Representación de protagonistas según su género en el E3 de 2019 de Anita Sarkeesiani.



Para ello, la adquisición de estas narrativas a la hora de implementarlas dentro de la elaboración de la experiencia gamificada es peligrosa por los sesgos que el propio medio tiene. 
El medio es en si mismo un generador de imaginarios sociales de desigualdad, ya que esta representación social de la realidad sirve para fomentar valores contrarios a los propuestos por el sistema educativo. Tal y como ha mencionado en este artículo, estos imaginarios surgen a través de la elaboración de esquemas mentales que toman forma a través de la imaginación. Un ejemplo de esta situación ocurre cuando pensamos en el personaje protagonista de cualquier obra conocida, ya que, si se utiliza el uso de la imaginación probablemente el protagonista de la obra que se tiene en mente sea un varón blanco de 30 o 40 años. Tal y como se puede apreciar con la imagen que se muestra a continuación, que reúne las características más comunes que presentan los personajes masculinos en las narrativas occidentales mainstream del momento.

Figura 2: Bingo sobre las principales características de los personajes masculinos en videojuegos de Brock Wilburii

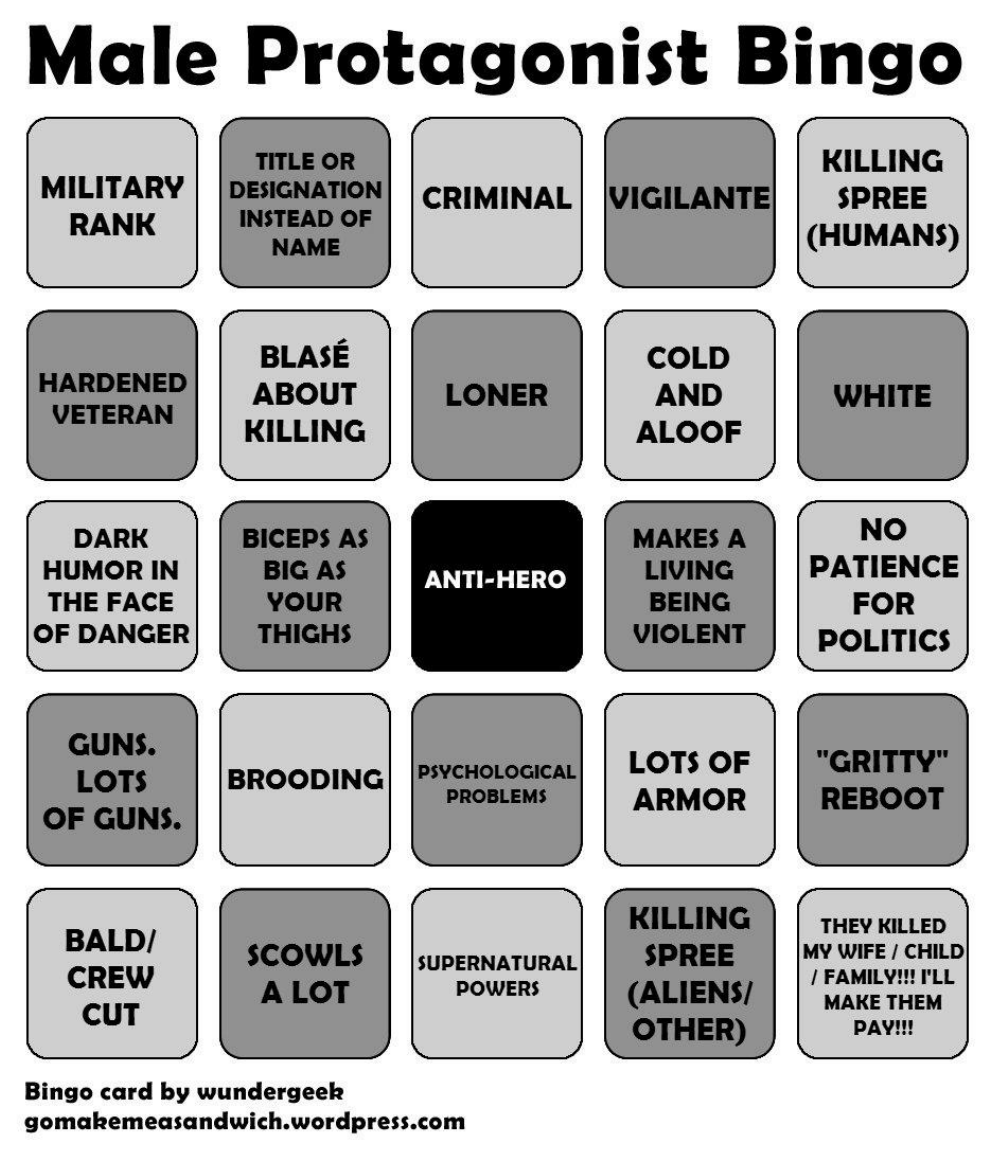

Una vez vista la forma de representación masculina (Figura 2) en los videojuegos el papel de los docentes consiste en establecer características contrarias a las mostradas en el bingo anterior y a su vez elaborar un proceso de empoderamiento del resto de grupos con la narrativa Revista RBBA $\mid$ Revista Binacional Brasil Argentina 
propuesta en el aula. Con este proceso se pueden lograr dinámicas que favorezcan el aprendizaje y lograr un espacio igualitario y sano dentro del aula.

\section{Conclusiones}

Está claro que esta metodología tiene sus beneficios y desventajas, pero tal y como hemos visto el currículo educativo se siente anticuado por la escasa adaptación a la realidad del momento y, por lo tanto, hay que tener en cuenta estas características antes de implementar esta metodología en el aula:

Los imaginarios sociales que surgen a través de esta perspectiva son negativos y crean desigualdad, es la labor docente la encarga de transformar este medio para obtener una experiencia sana y enriquecedora para todas las necesidades que presenta el alumnado.

Muchos de los procesos descritos con anterioridad requieren una serie de pasos previos a realizar para que el resultado final sea positivo y hay que justificar el uso de la gamificación con un propósito y si la parte del currículo de educación que se está a trabajar permite el uso de un sistema gamificado.

La adquisición de estas narrativas globalizadas tiene el riesgo de causar una construcción de la realidad ajena a la cercana y, por lo tanto, un distanciamiento del entorno cercano al alumnado.

Se produce una gran carga docente y responsabilidad en el profesorado, estas dinámicas favorecen la interacción del alumnado y la creación de experiencias, pero es el profesorado el responsable de lograr el funcionamiento de la actividad.

El contexto sociocultural del alumnado es diverso y muchas de las situaciones que experimentan en su día a día son muy diferentes a las que se encuentra en el aula, el uso de la gamificación puede proporcionar un entorno similar al suyo.

El sesgo de género dentro de estas obras es obvio y la elaboración de material docente sin este sesgo es necesario para poder mostrar otras posibilidades al alumnado.

Una vez vistas estas conclusiones tan solo queda poner en práctica lo expuesto anteriormente y así poder lograr un compendio de experiencias nuevas dentro del sistema educativo con el fin de innovar en la práctica docente y lograr que el alumnado adquiera de una mejor manera las enseñanzas necesarias para su formación y crecimiento como personas. 


\section{REFERENCIAS}

AARSETH, E. Doors and Perception: Fiction vs. Simulation in Games. Intermédialités / Intermediality, (9), 35-44. (2007). Disponible en: doi.org/10.7202/1005528ar.

BOGOST, I. "Why Gamification is Bullshit". 2014. Disponible en: bogost.com/writing/blog/gamification_is_bullshit Acceso en: 20 feb.2019.

CHARSKY, D. Characteristics From Edutainment to Serious Games: A Change in the Use of Game. Games and Culture. 2010. Disponible en: doi.org/10.1177/1555412009354727. Acceso en: 20 feb.2019.

CRAWFORD, G. et Muriel, D. Video games as Culture: considering the role and importance of videogames in contemporary society. Ed: Routledge Abingdin, Oxon: New York, 2018.

DETERDING, S., Dixon, D., Nacke, L. E., O'Hara, K. y Sicart, M. Gamification: Using gamedesign elements in non-gaming contexts. Conference on human factors in computing systems (pp. 2425-2428). New York: ACM. 2011. Disponible en: doi:10.1145/1979742.1979575. Acceso en: 20 feb.2019.

DETERDING, S., Dixon, D., Khaled, R. y Nacke, L. From game design elements to gamefulness: Defining "gamification". Proceedings of the 15th international academic MindTrek conference: Envisioning future media environments (pp. 9-15). 2011. New York: ACM. Disponible en: doi:10.1145/2181037.2181040. Acceso en: 20 feb.2019.

FUCHS, M; Fizek, S; Ruffino Paolo; Schrape, Niklas (editors). Rethinking Gamification. Lüneburg: Meson Press, 2014.

KAPP, K. M. The gamification of learning and instruction: Game-based methods and strategies for training and education. San Francisco: Pfeiffer, 2012.

KAPP, K. M. Gamification designs for instruction. en C. M. Reigeluth, B. J. Beatty \& R. D. Myers (Eds.), Instructional design theories and models (pp. 351-384). New York: Routledge, 2016. Disponible en: doi:10.4324/9781315795478. Acceso en: 20 feb.2019.

MORA, M. La teoría de las representaciones sociales de Serge Moscovici. Athenea Digital. 2002. Disponible en https://atheneadigital.net/article/view/n2-mora/55-pdf-es

LABRADOR, E. J. Sistemas gamificados mejorados a través de técnicas de experiencia de usuario. 2020, 203 f. Tesis (Doctorado en Tecnologies de la Informació i les Comunicacions i la seva gestió) Escola Tècnica Superior d'Engenyeria en Electrònica i Informàtica Engenyeria i Arquitectura La Salle, Universitat Ramon Llul, Barcelona.

LEE, J. J. \& Hammer, J. Gamification in Education: What, How, Why Bother? Academic Exchange Quarterly, 15(2). (2011) Disponible en https://www.researchgate.net/publication/258697764_Gamification_in_Education_What_Ho w_Why_Bother. Acceso en: 20 feb.2019.

PÉREZ, S. Situando los imaginarios sociales: aproximación y propuestas. Imagonautas. (2017) Disponible en http://imagonautas.webs.uvigo.gal/index.php /imagonautas/issue/viewIssue/28/31. Acceso en: 20 feb.2019.

PINTOS, J. L. Algunas precisiones sobre el concepto de imaginarios sociales. Revista Latina de Sociología. 2014.Disponible en: https://ruc.udc.es/dspace/handle/2183/14482. Acceso en: 20 feb.2019.

\begin{tabular}{l|l} 
Revista RBBA & Revista Binacional Brasil Argentina
\end{tabular} 
SCHUTZ, A. El problema de la realidad social. Buenos Aires: Ed: Amorrortu, 2015.

ZICHERMANN, G. y CUNNINGHAM, C. Gamification by design: Implementing game mechanics in web and mobile apps. Beijing: O'Reilly, 2011.

\section{SOBRE EL AUTOR}

Pablo Soto Casás es Estudiante de doctorado en el Programa de Marketing Político, Actores e Instituciones en Sociedades Contemporáneas por la Universidad de Santiago de Compostela (USC), España. Investigador del Centro de Investigación Feminista y estudios de género (CIFEX) de la USC. Dirección electrónica: pablo.soto.casas@rai.usc.es

ORCID 0000-0002-6653-8909

\section{Notas}

i Figura 1: Representación de protagonistas según su género en el E3 de 2019. Recuperado de: www.wired.com/story/e3-2019-female-representation-videogames/ Acceso: 31 mar 2020.

ii Figura 2: Bingo sobre las principales características de los personajes masculinos en videojuegos. Recuperado de:

www.rockpapershotgun.com/2018/04/08/lets-play-male-protagonist -bingo/ Acceso: 31 mar 2020.

Recebido em: 25/04/2020

Aprovado em: 01/06/2020

Publicado em: 01/07/2020 